\title{
High-speed, thermal damage-free ablation of brain tissue with femtosecond pulse bursts
}

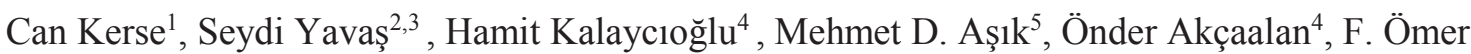 \\ Ilday ${ }^{1,2,4}$ \\ 1. Department of Electrical and Electronics Engineering, Bilkent University, Ankara, 06800, Turkey \\ 2. UNAM - Institute of Materials Science and Nanotechnology, Bilkent University, Ankara, 06800, Turkey \\ 3. FiberLAST, Inc., Ankara, 06531, Turkey \\ 4. Department of Physics, Bilkent University, Ankara, 06800, Turkey \\ 5. Nanotechnology and Nanomedicine Department, Institute of Science, Hacettepe University, Ankara, 06800, Turkey
}

\begin{abstract}
We report a novel ultrafast burst mode fiber laser system and results on ablation of rat brain tissue at rates approaching an order of magnitude improvement over previous reports, with no discernible thermal damage.
\end{abstract}

\section{INTRODUCTION}

Femtosecond pulses hold great promise for highprecision tissue removal. However, ablation rates are severely limited by the need to keep average laser power low to avoid collateral damage due to heat accumulation [1]. Furthermore, previously reported pulse energies preclude delivery in flexible fibers, hindering in vivo operation. Both of these problems can be addressed through use of groups of high-repetition-rate pulses, or bursts [2 - 3]. Here, we report a novel fiber laser and demonstrate ultrafast burst-mode ablation of brain tissue at rates approaching $1 \mathrm{~mm}^{3} / \mathrm{min}$, an order of magnitude improvement over previous reports. Burst mode operation is shown to be superior in terms of energy required and avoidance of thermal effects, compared to uniform repetition rates. These results can pave the way to in vivo operation at medically relevant speeds, delivered via flexible fibers to surgically hard-to-reach targets, or with simultaneous magnetic resonance imaging.

\section{MATERIALS AND METHODS}

The system produces up to $8 \mu \mathrm{J}$ pulses with $\sim 300 \mathrm{fs}$ FWHM pulse duration at $200 \mathrm{kHz}$ uniform pulse rep. rate or $22.3 \mathrm{MHz}$ intra-burst pulse rep. rate with $50 \mathrm{KHz}$ inter-burst repetition rate. Freshly harvested rat brain tissues, which were processed within an hour after dissection were used during the experiments, where we tested the effect of these two operating modes. The pulse energy on sample was kept at $3 \mu \mathrm{J}$. We performed raster scans over an area of $1 \mathrm{~mm} \times 1 \mathrm{~mm}$ with line separation of $2.5 \mu \mathrm{m}$ and scan speed of $250 \mathrm{~mm} / \mathrm{s}$ and with $80 \%$ duty cycle to avoid excessive heating. The raster scan was then repeated 50 times with total exposure time of
$80 \mathrm{~s}$. After irradiation, the samples were preserved in $4 \%$ paraformaldehyde solution, and then examined with a micro-CT scanner (Skyscan 1172, Bruker). For histological analyses, following fixation, the tissue samples were embedded into paraffin blocks and then cut into $5 \mu \mathrm{m}$ slices in sagittal plane, perpendicular to tissue surface. Then, slices were examined under light microscopy (Leica, DM 5000 B) following a standard

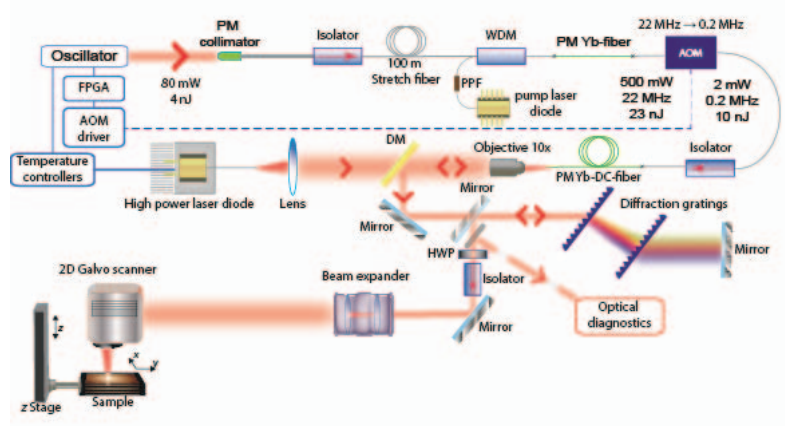

Fig. 1 - Schematic diagram of experimental setup for soft tissue processing. PM, polarization maintaining; PPF, pump protection filter; WDM, wavelength division multiplexer; DC, double clad; DM, dichroic mirror; HWP, half wave plate.

hemotoxylin-eosin staining procedure. The experimental setup is depicted in Fig. 1 including the laser stage and the material processing stage.

\section{RESULTS}

The temporal pulse train scheme of two operating modes, i.e. burst mode and uniform repetition rate mode is depicted in Fig. 2 (a). Whereas, in Fig. 2 (b-e), the 3D reconstructed micro-CT images and corresponding histological slices are depicted. The depth of the lesions on brain tissue were measured to be $\sim 1200 \mu \mathrm{m}$ and $\sim 1100 \mu \mathrm{m}$ for 4-pulsed burst and $200 \mathrm{kHz}$ uniform repetition rate modes, respectively. Our results indicate that it is possible to ablate at a rate of $\sim 1 \mathrm{~mm}^{3} / \mathrm{min}$ without any apparent heat damage to surrounding tissue. In contrast, earlier reports with solid state lasers utilized much higher energies and lower repetition rates. Using 

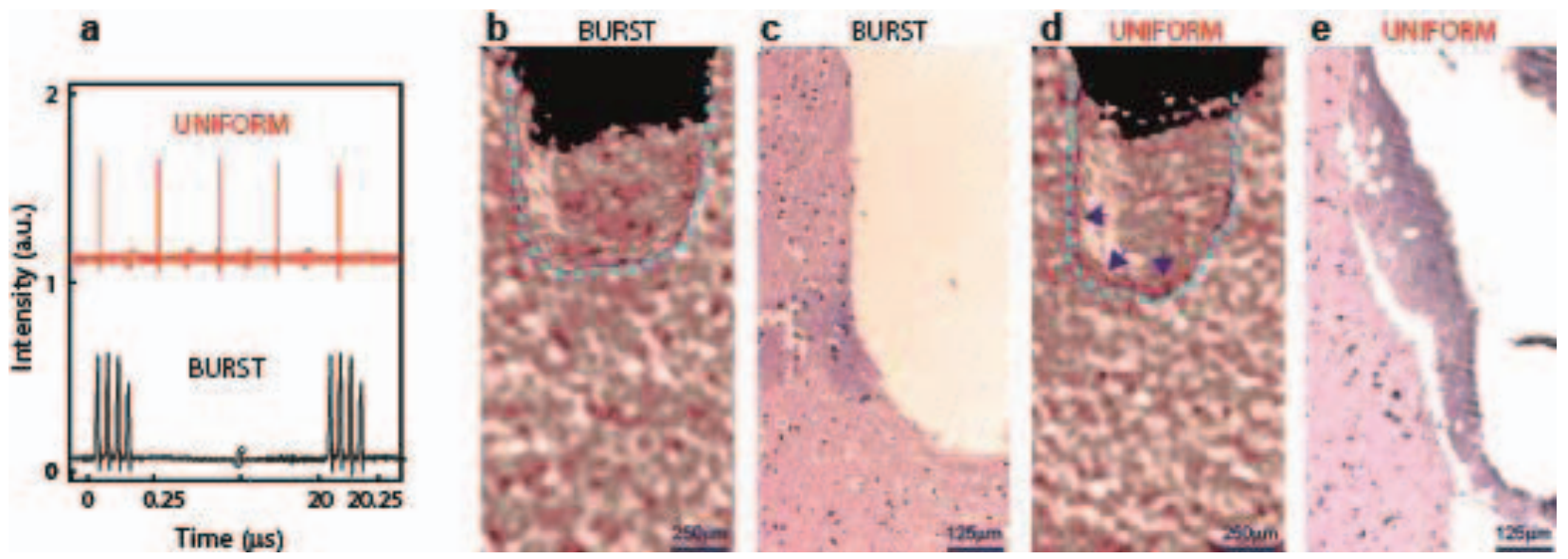

Fig. 2 - The temporal profile, micro-CT sections and histological appearances of burst and uniform mode operations. (a) Temporal profile of burst and uniform modes. (b) Micro-CT section of rat brain tissue sample ablated with burst mode. (c) Histological section of b. (d) Micro-CT section of rat brain tissue sample ablated with uniform mode. (e) Histological section of $\mathrm{d}$.

$165-\mu \mathrm{J}, 180-\mathrm{fs}$ pulses at $100 \mathrm{~Hz}$, the rate of ablation was reported as $0.09 \mathrm{~mm}^{3} / \mathrm{min}[4]$.

Even if the ablation depths are quite similar for both modes of operation, histological analysis reveals that there exists tremendous amount of heat affected zone for ablation with uniform repetition rate, as compared to burst mode. Burst application has minimized collateral damage to the tissue. On the other hand, uniform repetition rate application damaged cells. Also devascularization can be seen in Fig. 2 (e). Very little amount of tissue damage is observed burst application, which is localized to a specific area as seen in Fig. 2 (c). This might be due to the ablation scheme and improper focus adjustment. However, there is a prominent tissue loss and damage under uniform rate application site.

\section{CONCLUSION}

In conclusion, we report the first successful application of burst-mode femtosecond pulses to soft tissue using a unique fiber laser. The custom-developed fiber laser can operate either in uniform repetition rate pulse or ultrafast burst-mode regimes. Histological analysis confirmed that there was no discernible heatinduced damage in the surrounding area of the processed tissue. In contrast, histological analysis shows that the uniform repetition rate of operation of the laser under otherwise identical conditions resulted in substantial heat-induced damage. The burst-mode results represent nearly an order of magnitude improvement on previously reported ablation rates of brain tissue using solid-state lasers. The fiber laser parameters and burstmode operation explored in this work appear to be wellsuited to a variety of medical applications including neurosurgery and ophthalmology [5,6]. The pulse energy level during the processes is low enough that delivery within a flexible, single-mode, hollow-core fiber becomes a possibility as demonstrated recently for uniform repetition rate pulses in Ref. [7].

\section{ACKNOWLEDGMENT}

Can Kerse acknowledges full Ph.D. scholarship from TÜBITAK BIDEB 2211.

\section{REFERENCES}

[1] A. Vogel, J. Noack, G. Hüttman, and G. Paltauf, "Mechanisms of femtosecond laser nanosurgery of cells and tissues," Applied Physics B, vol. 81, no. 8, pp. 1015-1047, 2005.

[2] C. Kerse, H. Kalaycioğlu, O. F. Ilday, and E. Atalar, "Non-thermal Material and Tissue Processing with $100 \mathrm{MHz}$ and $500 \mathrm{MHz}$ Repetition Rate Bursts," CLEO Europe-OSA Technical Digest (CD)(Optical Society of America, 2013), paper CM-P. S, vol. 26, 2013.

[3] R. S. Marjoribanks, C. Dille, J. E. Schoenly, L. McKinney, A. Mordovanakis, P. Kaifosh, P. Forrester, Z. Qian, A. Covarrubias, Y. Feng, and others, "Ablation and thermal effects in treatment of hard and soft materials and biotissues using ultrafast-laser pulse-train bursts," Photon Lasers Med, vol. 1, no. 3, pp. 155-169, 2012.

[4] F. Loesel, J. Fischer, M. Götz, C. Horvath, T. Juhasz, F. Noack, N. Suhm, and J. Bille, "Non-thermal ablation of neural tissue with femtosecond laser pulses," Applied Physics B: Lasers and Optics, vol. 66, no. 1, pp. 121128, 1998.

[5] D. V. Palanker, M. S. Blumenkranz, D. Andersen, M. Wiltberger, G. Marcellino, P. Gooding, D. Angeley, G. Schuele, B. Woodley, M. Simoneau, and others, "Femtosecond laser-assisted cataract surgery with integrated optical coherence tomography," Sci Transl Med, vol. 2, no. 58, p. $58 \mathrm{ra} 85,2010$.

[6] P. S. Tsai, P. Blinder, B. J. Migliori, J. Neev, Y. Jin, J. A. Squier, and D. Kleinfeld, "Plasma-mediated ablation: an optical tool for submicrometer surgery on neuronal and vascular systems," Current opinion in biotechnology, vol. 20, no. 1, pp. 90-99, 2009.

[7] A. A. Lanin, I. V. Fedotov, D. A. Sidorov-Biryukov, L. V. Doronina-Amitonova, O. I. Ivashkina, M. A. Zots, C.-K. Sun, F. Omer Ilday, A. B. Fedotov, K. V. Anokhin, and others, "Air-guided photonic-crystal-fiber pulse-compression delivery of multimegawatt femtosecond laser output for nonlinear-optical imaging and neurosurgery," Applied Physics Letters, vol. 100, no. 10, pp. 101104-101104, 2012. 